\title{
Analysis of P67 Expression in Different Mouse Tissues
}

\author{
Bansidhar Datta", Rekha Datta \\ Department of Chemistry and Biochemistry, Kent State University, Kent \\ bdatta@kent.edu
}

\begin{abstract}
A \sim 1.9 \mathrm{~kb} c D N A$ containing the entire coding region, 5' UTR, and 3' UTR is cloned from rat tumor hepatoma (KRC-7) cells. It was further characterized for protein expression in rabbit reticulocyte lysates. Later, this cDNA was used for extensive mutagenesis and biochemical studies to identify specific domains/sites of rat p67 that are essential for its functions. To investigate whether similar size transcript is also present in mouse tissues, we examined the tissue distributions of mouse p67 transcript and protein from male and female mice. We found that there are two mouse p67 transcripts - one is $\sim 1.4 \mathrm{~kb}$ long whereas the other is $\sim 2.2 \mathrm{~kb}$ long. The $\sim 1.4 \mathrm{~kb}$ long transcript is present in almost all tissues tested whereas the $\sim 2.2 \mathrm{~kb}$ transcript is present in brain, spleen, skeletal muscle, and possibly in lungs. Our analysis for the tissue distributions of p67 protein detected several proteins corresponding to $\sim 67 \mathrm{kDa}, \sim 60 \mathrm{kDa}, 52 \mathrm{kDa}, 26 \mathrm{kDa}$, and possibly others in different proportions in various tissues of both male and female mice.
\end{abstract}

Keywords: mouse p67 gene, tissue distribution of mouse p67 transcript, tissue distribution of p67 in both male and female mice

Abbreviations used $k b$, kilobase pairs; p67, a $67 \mathrm{kDa}$ glycoprotein that binds to both eukaryotic initiation factor 2 (eIF2) and extracellular signal-regulated kinases 1 \& 2 (ERK1/2); p52, the downstream 108-480 segment of p67; and p26, the N-terminal 1-107 amino acid segment of p67.

\section{INTRODUCTION}

P67 is a cellular glycoprotein that runs around $67 \mathrm{kDa}$ region on a 15\% SDS-PAGE. It was first isolated as a eukaryotic initiation factor 2 (eIF2) - associated protein that blocks the phosphorylation of the smallest $\alpha$-subunit of eIF2 and regulates the rates of global protein synthesis (1). A $\sim 1.9 \mathrm{~kb}$ cDNA was cloned from rat tumor hepatoma cells and further characterized (2). Detailed mutagenesis studies combined with biochemical characterizations identified a series of domains/sites that are essential for p67's interactions with eIF2 subunits (3) and extracellular signal-regulated kinases (ERK1/2) (4) that modulates cell signaling to regulate cell growth, proliferation, differentiation, and apoptosis (5). P67 cDNA sequences and gene sequences from other species like yeast, plants, drosophila, mouse, and human have also been reported and their sequence conservations both at the open reading frames (ORFs) and promoter regions with rat p67 gene have been analyzed (for reviews see Refs. 6-8). This analysis also includes possible structural and functional conservations of rat p67 in part with heterologous systems like E. coli and parasites (6-8). In addition, chromosomal localizations of p67 true genes, pseudo genes, and exon/intron organizations from various organisms have also been reported (9). Based on these analyses we intended to believe that there might not be any isoform of p67 either at the mRNA level or at the protein level in different species. To verify this idea further, we performed both mRNA distribution and protein distribution of p67 in various mouse tissues. Our data show that a $\sim 1.4 \mathrm{~kb}$ long mRNA species of mouse p67 was detected in all tissues except testis, where it may have expressed in a very low level. In addition, another mRNA of $\sim 2.2 \mathrm{~kb}$ long is present in brain, spleen, skeletal muscle, and possibly in lungs. Several protein bands corresponding to molecular weights $\sim 67 \mathrm{kDa}, \sim 60 \mathrm{kDa}, 52 \mathrm{kDa}, 26 \mathrm{kDa}$, and possibly others are present in different proportions in various tissues of both male and female mice.

\section{Materials AND Methods}

All chemicals used in this study were obtained from Sigma Chemicals (St. Louis, MO), Merck (Darmstadt, Germany), ICN Biomedicals, Inc. (Aurora, Ohio), Fisher Chemicals (New Jersey), or GIBCO-BRL (Rockville, MD). All enzymes used in this study were purchased from New England Biolabs (Beverly, MA). Molecular mass markers were purchased from BioRad, and $\left(\gamma-{ }^{32} \mathrm{P}\right)$ ATP was purchased from Amersham. 


\subsection{Screening of Mouse Genomic Library}

DNA samples containing the full-length rat p67 cDNA and a BamHI and EcoRI fragment containing 900 bps that could be used as probes for Southern blots, were sent to Research Genetics, Huntsville, Alabama. Following company's standard procedures for screening mouse genomic library, ten positive clones were identified. These genomic DNA samples usually 100-160 kb long in YAC vector were put in E. coli and sent to us.

\subsection{Southern Blot Analysis}

E. coli cells harboring mouse p67 genomic DNA clones were grown in the presence of chloramphenicol for an extended period of time $(36 \mathrm{~h})$ at $30^{\circ} \mathrm{C}$, cells were harvested, and DNA samples were purified via Qiagen column chromatography. $10 \mu \mathrm{g}$ of these DNA samples were digested with $\mathrm{BamHI}$ at $37^{\circ} \mathrm{C}$ overnight. DNA samples were then analyzed on $0.6 \%$ agarose gels, transferred to nitrocellulose filters, and Southern blot analyses were performed following the procedures as described (10).

\subsection{Northern Blot Analysis}

A multiple-tissue Northern (MTN) blot was purchased from Clontech. The blot was processed for blocking, hybridization with $\left[{ }^{32} \mathrm{P}\right]$-labeled $\sim 400$ bp long cDNA from rat p67 (probe III, Fig. 1) or a beta-actin mRNA probe (from Clontech), and washing extensively with wash buffer followed by autoradiography using the procedures as described (10)

\subsection{Collection of Mouse Tissue Samples}

Adult $129 \mathrm{~Sv}$ mice (male and female) were purchased from Charles River and housed at the centralized facility (Department of Biological Sciences) at the University of Nebraska, Lincoln. Both male and female mice were dissected following the University's protocol. Various tissue samples were collected, immediately freezed in liquid nitrogen, and stored frozen until further analysis.

\subsection{Extraction of Total Protein Samples}

Frozen tissue samples were grinded into fine powders and lysis buffer $(25 \mathrm{mM}$ Tris- $\mathrm{HCl}, 100 \mathrm{mM}$ $\mathrm{KCl}, 0.5 \mathrm{mM}$ EDTA, $5 \mathrm{mM} \beta$-mercaptoethanol, and a cocktail of protease inhibitors containing PMSF, leupeptin, aprotinin, and vanadium orthophosphate) was added to resuspend the grinded tissue samples. After repeated freeze thaw cycles, the slurries were centrifuged at $12,000 \mathrm{rpm}$ and the supernatants were collected for total protein analysis. Protein concentrations were measured by BioRad assays following the protocols supplied by the company.

\subsection{Western Blot Analysis}

Equal amounts of total proteins samples from various tissues as indicated in legend to figures were analyzed for the levels of p67 in Western blots following the procedures as described (1).

\subsection{Antibodies}

Monoclonal antibody specific to $\alpha$ Actin was obtained from Sigma Chemicals and polyclonal antibodies specific to rabbit p67 were raised and characterized following the procedures as described (1).

\section{RESUlTS AND DisCUSSION}

To obtain the mouse p67 gene, the rat cDNA sample containing the entire p67 coding region, 5'UTR, and 3'UTR (total length $\sim 1.9 \mathrm{~kb}$ ) was sent out to Research Genetics (Huntsville, AL) for screening mouse (129/Sv strain) genomic pBAC library. Ten clones were obtained. The sizes of the DNA inserts in these clones vary from 60-165 kb. The genomic DNA samples from these clones were purified, digested overnight with EcoRI restriction enzyme, and analyzed by Southern blots (Fig. 1). Initially, the blot was probed with $\left[{ }^{32} \mathrm{P}\right]$-labeled probe I (Fig. 1C), later with probe II after stripping the probe I (Fig. 1B), and finally with probe III after removing the probe II (Fig. 1D). These three probes covered all eleven exons and ten introns, which are with varying lengths present in p67 gene (9). Among six clones analyzed in these experiments, clone 219G-10 (lanes 4 in all panels A-C) contained not only all the exons and introns of p67, it also had enough sequences both at the upstream 5' end and downstream 3'end of the p67 gene. We chose this genomic clone for further analysis. 


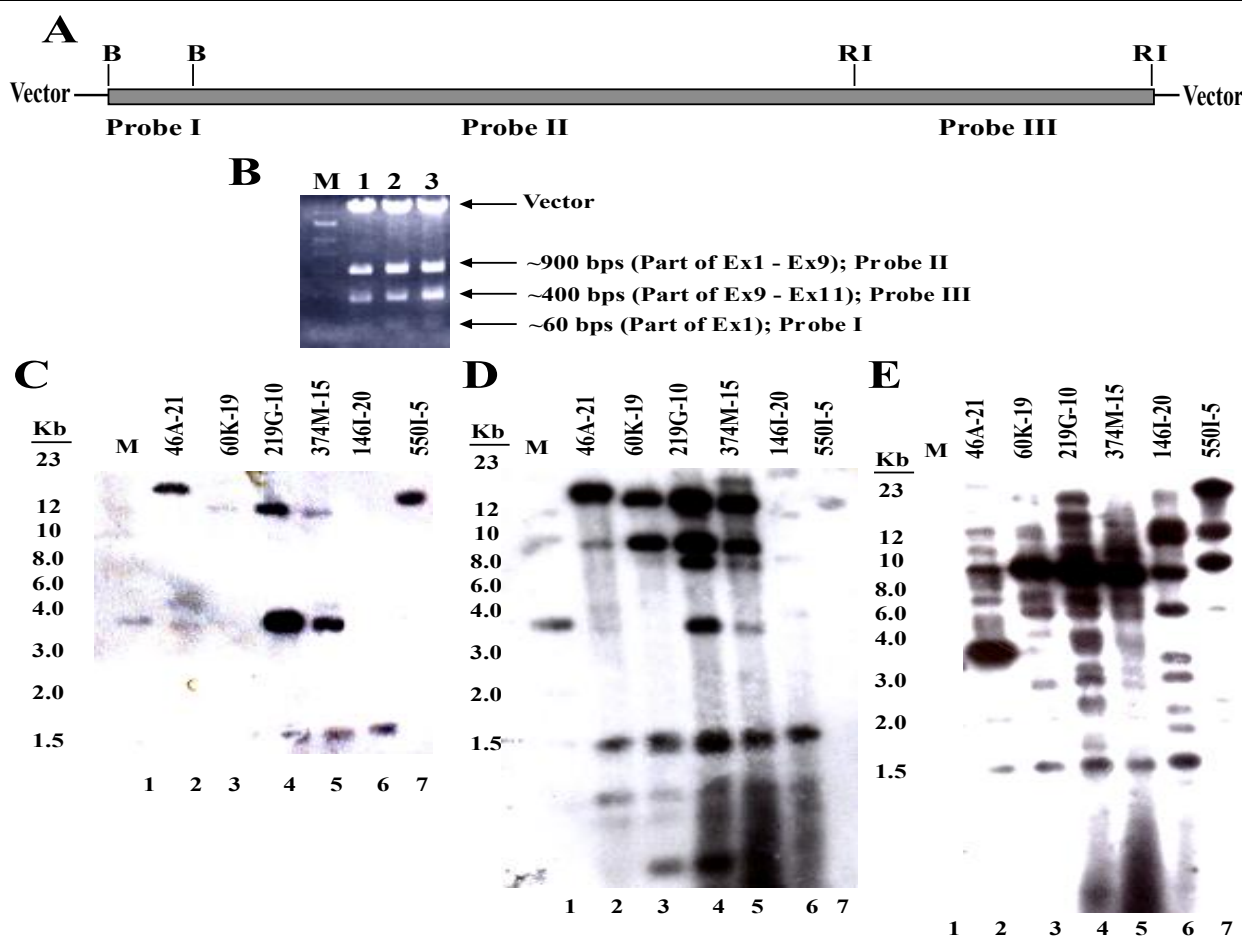

Fig1. Southern blot analysis of mouse p67 gene.(A) A schematic diagram of the entire p67-coding region from rat is shown. The restriction sites (B, BamHI; RI, EcoRI) are shown. The EGFP-p67 plasmid was digested with BamHI and EcoRI enzymes. The DNA fragments were analyzed on a $1 \%$ agarose gel and stained with ethidium bromide (B). The mouse genomic DNA samples from different clones were purified and digested with EcoRI at 37 degree $C$ overnight. The digested products were separated by $0.6 \%$ agarose gels, transferred to nitrocellulose filters, and analyzed by Southern blots using the labeled p67-specific cDNA probe I (C). Probe I from the blot was stripped off, and re-probed with probe II (D). Similarly, probe II was stripped off, and re-probed with labeled probe III (E). Digesting the entire p67-coding region with the BamHI and EcoRI restriction enzymes generated these probes. Probe I contains the nucleotide sequences from 1-60, probe II contains 61-960, and probe III contains the rest of the 3' end of the coding region. The positions of the size markers (in kb) are shown

First, we analyzed the mouse genomic DNA sequence database and used appropriate forward and reverse primers to pullout $\sim 1.5 \mathrm{~kb}$ long promoter region of mouse $\mathrm{p} 67$ gene. This promoter region was subcloned into M13mp18 for sequence verification and expression vectors pLuc and pCAT for the expression of luciferase and chloramphenicol acetyl transferase reporters respectively in mammalian cells. Detailed analyses including the length, nucleotide sequence, presence of various cis-elements, length of 3' UTR, and the start site in the context of the TATAA box were performed with the mouse p67 promoter and the results have recently been reported (9).

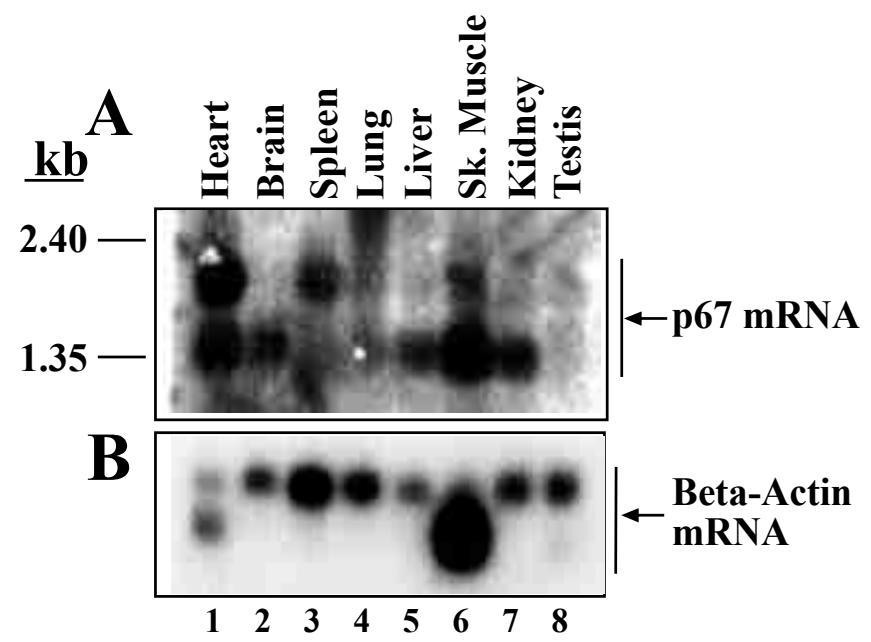

Fig2. Northern blot analysis of mouse p67 transcripts. A multiple-tissue Northern (MTN) blot (from Clontech) with 2 micro $g$ of poly $(A)^{+}$RNA samples from various tissues of adult 129Sv mice was analyzed by a Northern blot using ${ }^{32} P$-labeled rat $067 \mathrm{cDNA}$. The radiolabeled probe from the blot was stripped off and re-probed with $a^{32} P$-labeled probe from beta-actin $c D N A(B)$. Molecular markers in $k b$ are shown on the left 
Later, to check the distributions of mouse p67 transcript(s) we performed Northern blot analysis on a MTN Blot (Fig. 2). Our results show that there are at least two p67 transcripts. One of them is $\sim 1.4$ $\mathrm{kb}$ long, which includes 5'UTR, 3'UTR, and an ORF of unknown lengths and this is expressed ubiquitously in all tissues tested in these experiments. The second p67 transcript is $\sim 2.2 \mathrm{~kb}$ long and this is expressed predominantly in heart, spleen, skeletal muscle, and may be some extend in lungs (Fig. 2A, lanes 1, 3, 4, and 6). The cloned rat p67 cDNA (2) is about the same size as the second transcript of mouse p67. Detection of p67 transcripts in mouse testis seems to be inconclusive because we do not see any transcript(s) in this tissue but p67 protein is quite abundant (see later). Due to the pretty uneven distributions of control beta-actin mRNA in the same Northern blot (Fig. 2B), we however cannot predict the relative abundances of the transcripts of mouse p67 in various mouse tissues tested in these experiments. Nonetheless, results from these experiments definitely allow us to conclude that mouse p67 gene expresses two different transcripts with varying lengths. It is therefore a challenge to identify the proteins corresponding to these p67 transcripts.

As a first step to our challenges we collected both male and female mice tissue samples. As shown in Figure 2, Clontech Company also used identical tissues to prepare the MTN Blot that was used for Northern blotting experiments. Total protein samples were prepared from the frozen tissues and analyzed on Western blots (Figs $3 \& 4$ ). Our results show that a protein $\sim 67 \mathrm{kDa}$ size in $15 \%$ SDSPAGE is ubiquitously present in all tissues from both male and female mice tested in these experiments (Figs 3A \& 4A), although, its relative amounts in these tissues may vary slightly. Another protein $\sim 60 \mathrm{kDa}$ size is present in all tissues used from both male and female mice for these experiments except brain and liver of both male and female mice possibly express this protein in extremely low level (compare lanes $1,3-4, \& 6-8$ with lanes $2 \& 5$ ). The relative ratios of the $\sim 67 \mathrm{kDa}$ and $\sim 60 \mathrm{kDa}$ proteins in tissues they are expressed also vary widely. At present, we do not have any clue whether these proteins - $\sim 67 \mathrm{kDa}$ and $\sim 60 \mathrm{kDa}$ sizes correspond to the mouse p67 transcripts $\sim 2.2 \mathrm{~kb}$ and $\sim 1.4 \mathrm{~kb}$ long respectively.

In our Western blotting experiments we have also detected some faster migrating proteins running around $52 \mathrm{kDa}$ (or p52), $26 \mathrm{kDa}$ (or p26), and others with lower molecular weights in some tissues. For example in male mice, p52 is detected in tissues like brain, liver, skeletal muscle, kidney, and testis in varying amounts (Fig. 3A, lanes 2, 5-8). Similarly, p26 is detected almost in all tissues tested except spleen and testis where its abundance may be very low (Fig. 3A, lanes 3 and 8). Another faster migrating lower molecular weight protein running around $\sim 15-20 \mathrm{kDa}$ size region on SDS-PAGE is also detected almost in all tested male tissues except in skeletal muscle and testis where it may present in extremely low level (Fig. 3A, lanes $6 \& 8$ ). In female mice tissues, the relative presence of the faster migrating proteins like p52, p26, and others varies quite a bit. For example, both p52 and p26 proteins are present in all female mouse tissues tested in the experiments but their levels are very low as compared to similar male mice tissues (compare lanes 1-8 in Fig. 3A with lanes 1-8 in Fig. 4A).

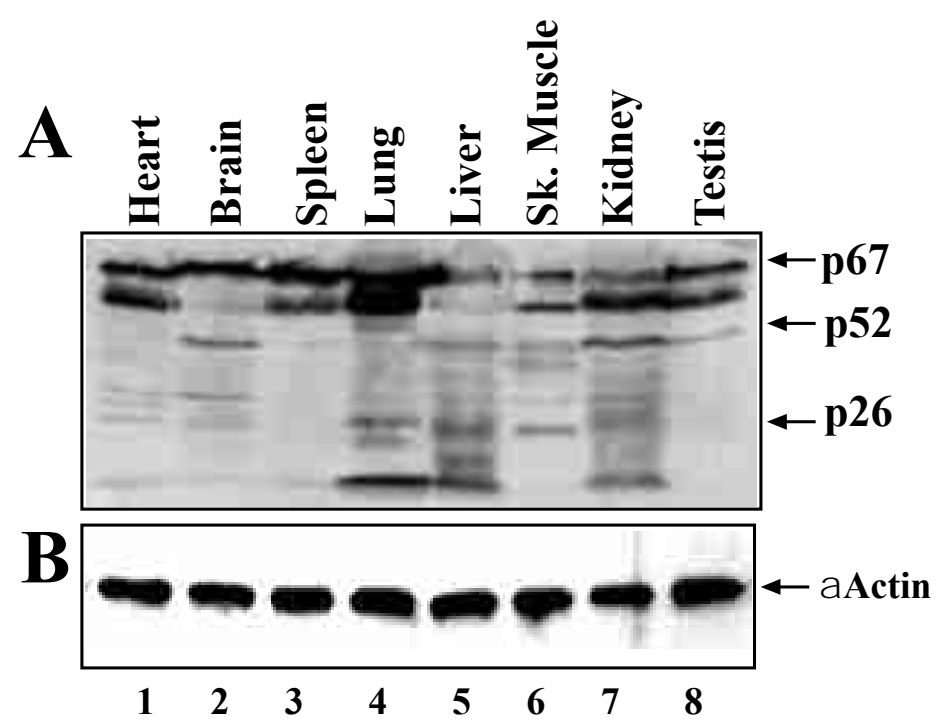

Fig3. Immunoblot analysis of mouse p67 in multiple tissues from male mice. Samples from different tissues as indicated were collected by dissecting male 129 Sv mice and immediately frozen in liquid nitrogen. Total protein extracts were obtained from frozen tissues and $100 \mu \mathrm{g}$ of total proteins from each tissue were analyzed on a Western blot using p67 polyclonal antibodies (A). For loading control, same amounts of total protein samples 
from different tissues were analyzed on Western blot and probed with monoclonal antibody specific to $\alpha$ Actin (B). Full-length p67, its 108-480 amino acid segment, and its N-terminal 1-107 amino acid segment were indicated as p67, p52, and p26 respectively. The amount of protein samples and the integrity of their transfer to nitrocellulose filters were verified by staining with Ponceau-S solution.

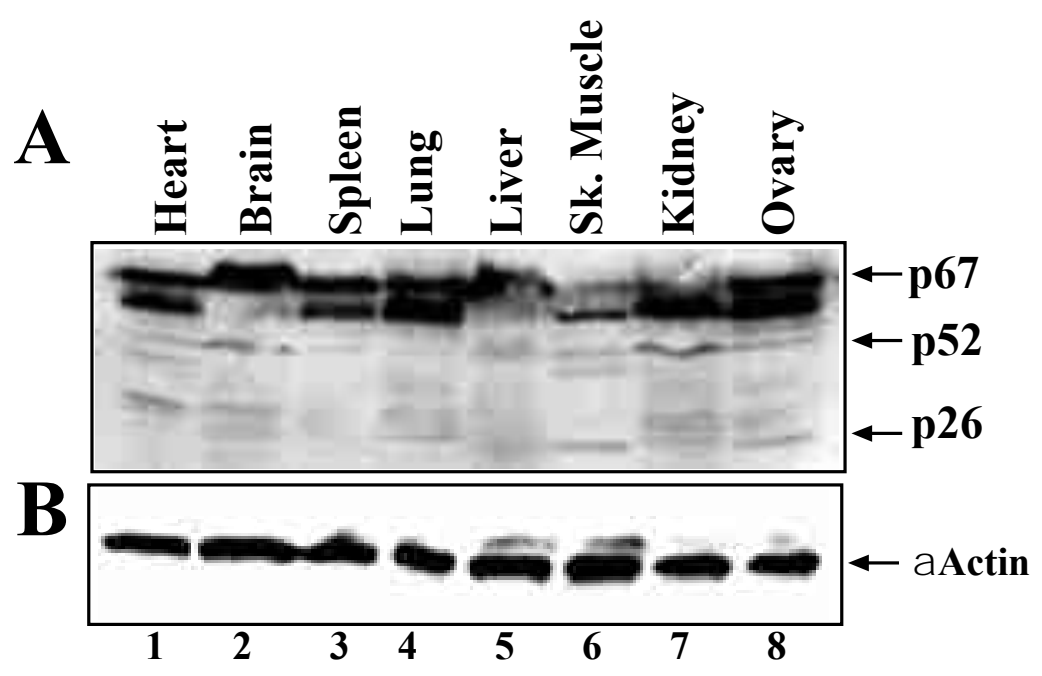

Fig4. Immunoblot analysis of mouse p67 in multiple tissues from female mice. Similar Western blot analyses were performed as mentioned in legend to Figure 3 taking the total protein samples from various tissues of female $129 \mathrm{~Sv}$ mice. Lysates from the indicated tissues (100 $\mu \mathrm{g}$ of protein equivalent) were loaded in each lane. Expressions of full-length $\mathrm{p} 67$ and its segments were assessed by immunoblot analysis with an anti-p67 antibody (A). For loading control, similar Western blot analysis was performed and probed the blot with anti- $\alpha$ Actin antibody $(B)$. The amount of protein and the integrity of transfer were verified by staining with Ponceau-S solution.

Our previous studies on structure-function relationships of rat p67 revealed several important facts (for reviews see Refs. 6-8). Among these notable findings the most important fact is that rat p67 has auto-proteolysis activity and this activity generates two major p67 fragments - p26, the N-terminal 1107 amino acid residues long and p52, which contains the downstream 108-480 amino acid residues of p67 (11). We have demonstrated these facts both in vitro and in vivo (Ref. 11 and the supplemental studies therein). In addition, we have also reported that p52 segment of rat p67 alone has autoproteolysis activity at least in vitro (11). Recently, Clinkinbeard et. al., reported that human p67 also has auto-proteolysis activity that generates p52 segment of this protein and this activity is further augmented by a metalloproteinase, calpain (12). We therefore believe that the faster migrating proteins like p52 and p26 are the cleavage products of full-length p67, which may have originated from $\sim 2.2 \mathrm{~kb}$ transcript whereas, $\sim 60 \mathrm{kDa}$ protein may coming from the translation of $\sim 1.4 \mathrm{~kb}$ transcript from mice. Either p52 or p26 or both may also have originated from the later transcript possibly translating into a $\sim 60 \mathrm{kDa}$ protein but it may be very unlikely. Because the full-length $\sim 1.4$ $\mathrm{kb}$ transcript can be translated into a polypeptide containing $\sim 400$ amino acid residues with a minimum length of 5' UTR and 3' UTR. Obviously, more detailed studies are required to clone and characterize p67 transcripts from mouse and possibly other organisms to identify transcript-specific proteins of p67 from various species including mouse.

\section{REFERENCES}

[1] Datta, B., Chakrabarti, D., Roy, A.L., and Gupta, N.K. (1988) Roles of a 67kDa polypeptide in protein synthesis inhibition in heme-deficient reticulocyte lysates. Proc. Natl. Acad. Sci. USA 85, 3324-3328.

[2] Wu, S., Gupta, S., Chatterjee, N., Hileman, R.E., Kinzy, T.G., Denslow, N.D., Merrick, W.C., Chakrabarti, D., Osterman, J.C., and Gupta, N.K. (1993) Cloning and characterization of complementary DNA encoding the eukaryotic initiation factor 2-associated 67-kDa protein $\left(\mathrm{p}^{67}\right)$ J. Biol. Chem. 268, 10796-10801.

[3] Ghosh, A., Datta, R., Majumdar, A., Bhattacharya, M., and Datta, B. (2006) The N-terminal lysine residue-rich domain II and the 340-430 amino acid segment of eukaryotic initiation factor 2 -associated glycoprotein p67 are the binding sites for the $\gamma$-subunit of eIF2. Exp. Cell Res. 312, 3184-3202. 
[4] Majumdar, A., Ghosh, A., Datta, S., Prudner, B., and Datta, B. (2010) P67/MetAP2 suppresses K-RasV12 mediated transformation of NIH3T3 mouse fibroblasts in culture and in athymic mice. Biochemistry 49,10146-10157.

[5] Meloche, S., and Pouyssegur, J. (2007) The ERK1/2 mitogen-activated protein kinase pathway as a master regulator of the G1- to S-phase transition. Oncogene 26, 3227-3239.

[6] Datta, B. (2000) MAPs and POEP of the roads from prokaryotic to eukaryotic kingdoms. Biochimie 82, 95-107.

[7] Datta, B. (2009) Roles of P67/MetAP2 as a tumor suppressor - a review. Biochim. Biophys. Acta 1796, 281-292.

[8] Datta, B. (2015) Diversified roles of p67/MetAP2 as a regulator of cell growth and differentiation, in tumor suppression, and in obesity. Review, Curr. Topics Biochem. Res. 16, 41-52.

[9] Datta, B., Earl, D., Roods, M., Datta, S. (2014) Analysis of p67/MetAP2 gene from mouse, rat, and human. Intl. J. Mol. Genet._5, 1-12.

[10] Ausubel, F. M., Brent R., Kingston R. E., Moore D. D., Seidman J. G., Smith J. A., Struhl K. (1992) Current Protocols in Molecular Biology, Vol. 1, section 8.1.

[11] Datta, B., Ghosh, A., Majumdar, A., and Datta, R. (2007) Autoproteolysis of rat p67 generates several peptide fragments: the N-terminal fragment, p26, is required for the protection of eIF $2 \alpha$ from phosphorylation. Biochemistry 46, 3465-3475.

[12] Clinkinbeard, T., Ghoshal, S., Craddock, S., Pettigrew, L. C., Guttmann, R. P. (2013) Calpain cleaves methionine aminopeptidase-2 in a rat model of ischemia/reperfusion, Brain Res. 1499, 129-135. 